\title{
Expression and structural characterization of peripherin/RDS, a membrane protein implicated in photoreceptor outer segment morphology
}

\author{
Werner Louwrens Vos • Sebastian Vaughan • \\ Patrick Y. Lall • John G. McCaffrey • \\ Monika Wysocka-Kapcinska · John B. C. Findlay
}

Received: 11 June 2009/Revised: 1 October 2009/Accepted: 9 October 2009/Published online: 18 November 2009

(C) European Biophysical Societies' Association 2009

\begin{abstract}
Peripherin/RDS is a member of the tetraspanin family of integral membrane proteins and plays a major role in the morphology of photoreceptor outer segments. Peripherin/RDS has a long extracellular loop (hereafter referred to as the LEL domain), which is vital for its function. Point mutations in the LEL domain often lead to impaired photoreceptor formation and function, making peripherin/RDS an important drug target. Being a eukaryotic membrane protein, acquiring sufficient peripherin/ RDS for biophysical characterisation represents a significant challenge. Here, we describe the expression and characterisation of peripherin/RDS in Drosophila melangolaster Schneider (S2) insect cells and in the methylotrophic yeast Pichia pastoris. The wild-type peripherin/RDS and the retinitis pigmentosa causing $\mathrm{P} 216 \mathrm{~L}$ mutant from $\mathrm{S} 2$ cells are characterised using circular dichroism (CD) spectroscopy. The structure of peripherin/RDS and of a pathogenic mutant is assessed
\end{abstract}

The more you see: spectroscopy in molecular biophysics.

W. L. Vos · M. Wysocka-Kapcinska - J. B. C. Findlay ( ()

The Marie Curie Laboratory for Membrane Proteins, National

University of Ireland Maynooth, County Kildare, Ireland

e-mail: john.findlay@nuim.ie

W. L. Vos

e-mail:wessel.vos@nuim.ie

P. Y. Lall · J. G. McCaffrey

Department of Chemistry, National University of Ireland

Maynooth, County Kildare, Ireland

S. Vaughan · J. B. C. Findlay

Institute for Membrane and Systems Biology,

Faculty of Biological Sciences, University of Leeds,

Leeds LS2 9JT, United Kingdom spectroscopically for the first time. These findings are evaluated in relation to a three-dimensional model of the functionally important LEL domain obtained by protein threading.

Keywords Pichia - S2 cells - Circular dichroism . Protein threading $\cdot$ Peripherin-2 $\cdot$ Retinitis pigmentosa

$\begin{array}{ll}\text { Abbreviations } \\ \text { LEL domain } & \text { Long extracellular loop domain } \\ \text { CD } & \text { Circular dichroism } \\ \text { p/RDS } & \text { Peripherin/retinal degeneration slow } \\ \text { NMR } & \text { Nuclear magnetic resonance } \\ \text { CD81 } & \text { Cluster of differentiation } 81 \\ \text { DDM } & n \text {-Dodecyl beta-D-maltoside } \\ \text { BCA } & \text { Bicinchoninic acid } \\ \text { LC-MS/MS } & \text { Liquid chromatography-mass } \\ & \text { spectrometry/mass spectrometry } \\ \text { ROM-1 } & \text { Retinal outer segment membrane protein 1 } \\ \text { FTIR } & \text { Fourier transform infrared } \\ \text { SDS } & \text { Sodium dodecyl sulfate } \\ \text { PAGE } & \text { Polyacrylamide gel electrophoresis }\end{array}$

\section{Introduction}

Tetraspanins are an important mammalian membrane protein family that is involved in roles such as the immunological response (Levy et al. 1998), sperm-egg fusion (Hemler 2003) and vision (Molday et al. 1987). Also, tetraspanins have been shown to be implicated in various diseases, including cancer (Lazo 2007), hepatitis C (Zhang et al. 2004) and retinitis pigmentosa (Shastry 
a

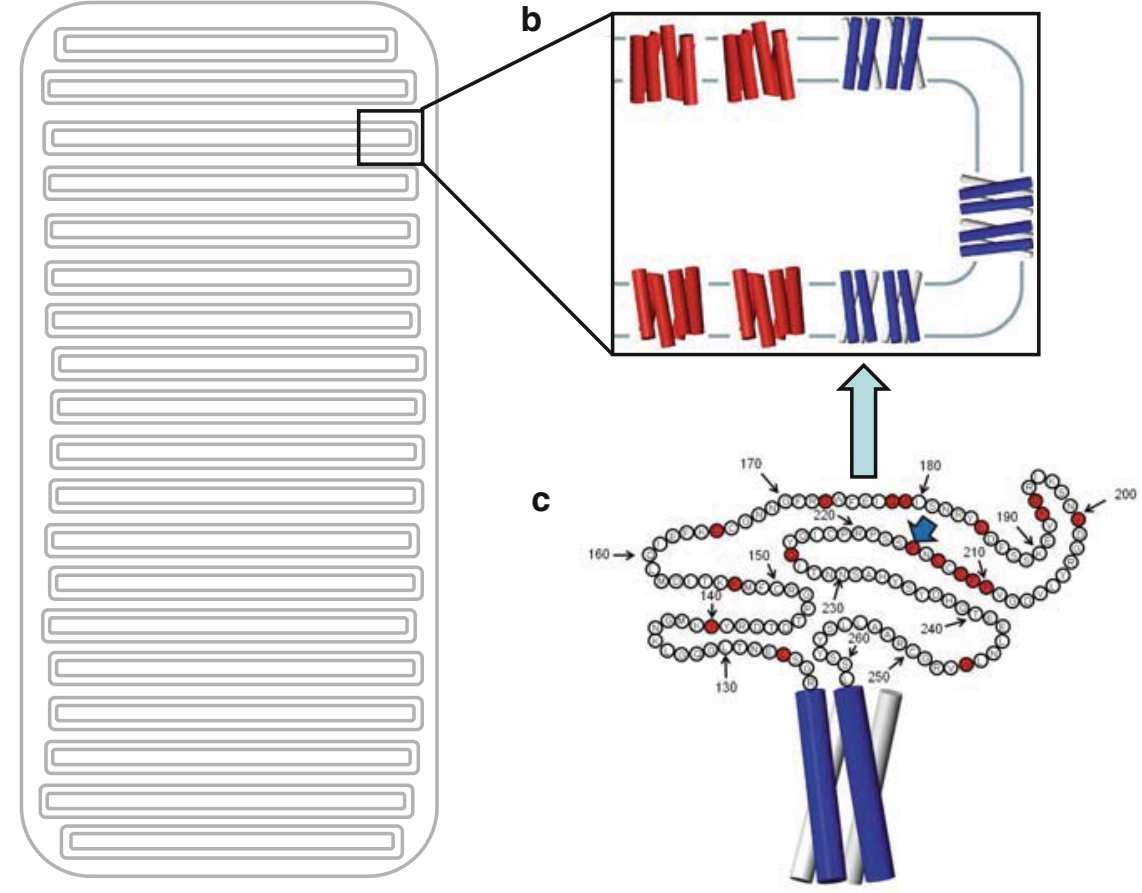

Fig. 1 a Schematic representation of a rod cell outer segment containing flattened lipid vesicles. b Schematic magnification of the rim region of the flattened vesicles containing rhodopsin (transmembrane helices are red cylinders, orientation taken from protein databank file 1JGJ) and peripherin rds (transmembrane helices are blue and white cylinders, orientation taken from protein databank file 2AVZ, a modeled structure of tetraspanin CD81). To prevent crowding, only two p/RDS molecules in the tetrameric core complex

1994). Although their exact function remains elusive, tetraspanins are known to be the key components in the 'tetraspanin web', protein-enriched microdomains reminiscent of lipid rafts [for a review, see (Hemler 2005)]. In this web, tetraspanins are thought to act as 'molecular organisers' or 'scaffolding proteins'. Being eukaryotic membrane proteins, obtaining sufficient quantities of tetraspanin protein for structural characterisation can be a limiting factor for structural studies. The only tetraspanin that has been characterised structurally is CD81, which was expressed in high levels $(1.75 \mathrm{mg} / \mathrm{l}$ culture $)$ in the methylotrophic yeast Pichia pastoris (Jamshad et al. 2008) and in HEK293S-TetR-inducible cell lines (Takayama et al. 2008) at a final concentration of $\sim 26 \mu \mathrm{g} / 3 \times 10^{7}$ cells per $15 \mathrm{~cm}$ tissue culture plate. The functionally important large extracellular loop (hereafter referred to as LEL domain) of CD81 has been overexpressed in $E$. coli as a separate, water-soluble protein, which enabled the determination of a high-resolution X-ray structure (Kitadokoro et al. 2001).

Here, we present our recent studies on the expression and spectroscopic characterisation of recombinant peripherin/RDS (hereafter referred to as $\mathrm{p} / \mathrm{RDS}$ ). $\mathrm{P} / \mathrm{RDS}$ is are shown, and the loops are not shown. c Topology model of peripherin/RDS based on glycosylation scanning from earlier work. TM helices 3 and 4 are indicated in blue; helices 1 and 2 are indicated in white. The large extracellular loop 2 (LEL domain) is given as a string of circles, with inside the one-letter abbreviation for the amino acid residues. Mutations implicated in autosomal dominant retinitis pigmentosa are highlighted in red; the $\mathrm{P} 216 \mathrm{~L}$ mutation is indicated with the blue arrow

implicated in many hereditary diseases that lead to impaired vision, most notably autosomal dominant retinitis pigmentosa. The majority of the pathogenic mutations are single-point mutations in the LEL domain (see Fig. 1: the amino acid residues in the LEL domain are indicated explicitly with their one-letter codes). In vivo, p/RDS is located in the outer segment of rod cells, where it is involved in the flattening of the membrane discs (Wrigley et al. 2000) that contain rhodopsin, the protein responsible for the initial events in phototransduction [i.e., absorption of a photon and initiation of the biochemical pathway that ultimately lead to an hyperpolarisation of the rod cell outer membrane; for a review see (Palczewski 2006)]. P/RDS has the hallmark characteristics of a tetraspanin: i.e., four transmembrane $\alpha$-helices and an LEL domain with the CysCys-Gly motif that sets it apart from other proteins with four transmembrane $\alpha$-helices (Wrigley et al. 2002). The main difference between $\mathrm{p} / \mathrm{RDS}$ and other tetraspanins is the size of the LEL domain, which is almost twice as long as that of other tetraspanins such as CD81 (140 vs. 87 amino acid residues). This characteristic is of particular interest since most pathogenic mutations are located in the p/RDS LEL-domain. 
In this paper, for the first time, we demonstrate expression of $\mathrm{p} / \mathrm{RDS}$ in quantities sufficient for spectroscopic characterisation. The protein is expressed in two different expression systems, cultured insect cells ( $\mathrm{S} 2$ cells) and the methylotrophic yeast Pichia Pastoris. Both the wild-type protein and the pathogenic L216P mutant expressed in S2 cells have been characterised using SDSPAGE and CD spectroscopy. The results suggest that the structural disturbance of the LEL domain due to the pathogenic point mutation is small. Interestingly, our CD data suggest that the structure of the p/RDS LEL domain is different from that of other tetraspanins at least in the additional protein fragment, offering new insights on the functional role of $\mathrm{p} / \mathrm{RDS}$ in disk morphogenesis.

\section{Methodology}

\section{Molecular biology}

Peripherin/retinal degeneration slow cDNA was a kind gift of Prof. Jane Farrar (Trinity College, Dublin). The p/RDS gene was cloned into the pAC5.1-V5-HIS A expression vector using standard molecular biology techniques (see Fig. 2 for the resulting constructs). The P216L mutant $\mathrm{p} / \mathrm{RDS}$ was prepared with the QuikChange site-directed mutagenesis kit using the original cDNA as a template and cloned into the pAC5.1-V5-HIS expression vector. The $\mathrm{p} / \mathrm{RDS}$ gene was subcloned from the pAC5.1-V5-HIS A

\section{a}

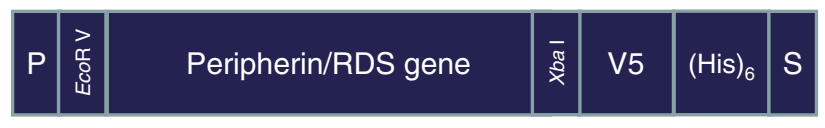

b

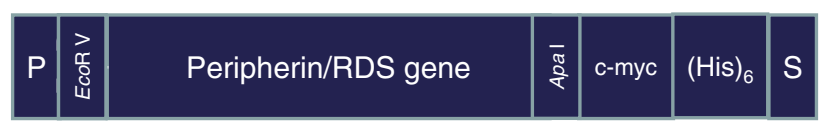

Fig. 2 a DNA construct used for the expression of recombinant p/RDS in S2 cells. P is the promotor for expression of the Drosophila actin $5 \mathrm{c}$, which permits high-level constitutive expression of heterologous proteins. EcoR V and Xba I are the restriction sites used for cloning the of the PCR product into the Drosophila pAc5.1/V5-His expression vector. V5 is the V5 epitope that is used for purification and identification of the protein. (His) ${ }_{6}$ is the hexahistidine tag that is used in the purification of the protein. $\mathrm{S}$ is the stop codon on the expression vector. b DNA construct used for the expression of recombinant $\mathrm{p} / \mathrm{RDS}$ in Pichia pastoris. $\mathrm{P}$ is the AOX1 promotor that allows methanol-induced, high-level expression in P. pastoris. EcoR I and Apa I are the restriction sites that were used to subclone the p/RDS gene from the Drosophila expression vector into the pPICZ, A expression vector for $P$. pastoris. c-myc is the c-myc epitope that was used for identification of the protein. (His) ${ }_{6}$ is the hexahistidine tag that was used for purification of the protein. $\mathrm{S}$ is the stop codon on the expression vector vector into the Pichia Pastoris expression vector pPICZ A using the Eco RI and Apa I restriction sites. The nucleotide sequence of the final expression vectors was confirmed by Lark technologies using automated DNA sequencing.

Protein expression and purification

Chemicals were purchased from Sigma unless specified otherwise. S2 cells were transfected using the calcium phosphate protocol for stable cell lines [version H (Invitrogen 2003)]. Cultures were expanded to a final volume of $300 \mathrm{ml}$ in a $\sim 2-1$ spinner flask (Jencons technologies) where they were grown to a cell density of $1-3 \times 10^{7}$ cells $/ \mathrm{ml}$. Cells were harvested and stored at $-80^{\circ} \mathrm{C}$ prior to subsequent processing. For purification, the pooled fractions were lysed on ice osmotically using $20 \mathrm{mM}$ Tris, $\mathrm{pH}$ 7.4 , in the presence of complete EDTA-free protease inhibitor (Roche). Lysed cells were collected by centrifugation at 45,000 rpm in a Beckman TL-100 ultracentrifuge for $1 \mathrm{~h}$ at $4^{\circ} \mathrm{C}$. Membrane proteins were extracted from the lysed cells using $1 \%$ DDM in $50 \mathrm{mM} \mathrm{NaH} \mathrm{PO}_{4}, \mathrm{pH} 8.0$ (Gibco), in the presence of complete protease inhibitor for $1 \mathrm{~h}$ at room temperature with agitation. Cell debris and insoluble protein were removed by centrifugation for $1 \mathrm{~h}$ at $4^{\circ} \mathrm{C}$ at 45,000 rpm in a Beckman TL-100 ultracentrifuge. The first purification step was carried out with pre-equilibrated Ni-NTA superflow (Qiagen) using a disposable column (Pierce chemicals). The second purification step was performed using V5-antibody agarose (Abcam) preequilibrated with Ni-NTA elution buffer (1\% DDM, $50 \mathrm{mM} \mathrm{NaH}{ }_{2} \mathrm{PO}_{4}, 300 \mathrm{mM} \mathrm{NaCl}, 250 \mathrm{mM}$ imidazole, $\mathrm{pH}$ 8.0). Protein was eluted from the $\mathrm{V} 5$ agarose by addition of V5-antibody elution buffer (2.5 mM glycine $\mathrm{pH} 2.0$ ); $100 \mu \mathrm{l}$ Tris- $\mathrm{HCl}$ was added immediately to the $1-\mathrm{ml}$ elution fractions to adjust the $\mathrm{pH}$ to 8.0 immediately after elution. HRP-conjugated V5 antibody was purchased from Invitrogen.

Pichia pastoris cells (strain KM71H) were transformed with a PmeI linearised p/RDS pPICZ expression vector. Transformation was performed using the lithium method described in the Invitrogen manual [Version D (Invitrogen 1997)]; the largest colonies were selected for subsequent analysis. Integration of the $\mathrm{p} / \mathrm{RDS}$ gene into the genome was confirmed using the PCR method described in the manual. Stably transformed cells were spread on YPD plates [1\% yeast extract, $2 \%$ peptone (BD), $2 \%$ glucose, $2 \%$ agar] with media containing $100 \mu \mathrm{g} / \mathrm{ml}$ zeocin. After 3 days, cells were scraped from the YPD plates and diluted into YP medium in the presence of $2 \%$ glycerol. Cells were grown overnight until the absorbance at $600 \mathrm{~nm}$ reached $\sim 5$ the following morning. Cells were harvested by centrifugation at $7,000 \mathrm{~g}$ for $5 \mathrm{~min}$ at $25^{\circ} \mathrm{C}$ and diluted by a factor of 5 into YP medium. Methanol was added to a final 

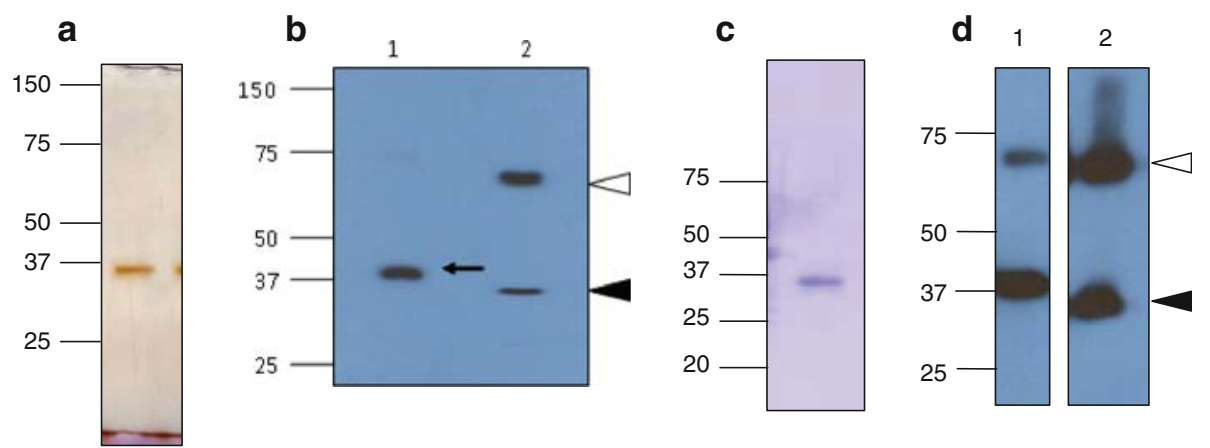

Fig. 3 a Silver-stained SDS gel of purified p/RDS from S2 cells using subsequently Ni-NTA superflow agarose and V5-agorose. b Western blot of p/RDS from S2 cells in the presence 1 and in the absence 2 of reducing agent, respectively. The blot was probed with monoclonal anti-V5 antibody. Note that the blot was performed using the solubilised membrane protein fraction, described in Materials and

concentration of $1 \%$, and cells were induced for $8 \mathrm{~h}$, after which they were harvested and stored at $-80^{\circ}$ until purification. Each cell pellet (the equivalent of $50 \mathrm{ml}$ of culture) was resuspended in $1 \mathrm{ml}$ of lysis buffer (PBS tablets from Gibco: $10 \mathrm{mM} \mathrm{NaPO}_{4}, 150 \mathrm{mM} \mathrm{NaCl}, \mathrm{pH}$ 7.4). An equal volume of acid-washed glass beads (425-600 nm, 30-40-US sieve) was added to the cell suspension; cells were lysed in a $15-\mathrm{ml}$ plastic tube by 10 times $30 \mathrm{~s}$ vigorous vortexing on a IKA Genius 3 vortex at full power with 30-s intervals, during which the cells were kept on ice. The lysate was centrifuged for $1 \mathrm{~min}$ at 7,000 $\mathrm{g}$ to remove cell debris. Cell membranes in the supernatant were collected by centrifugation at 45,000 rpm in a Beckman TL-100 ultracentrifuge for $1 \mathrm{~h}$ at $4^{\circ} \mathrm{C}$. The supernatant was discarded, and the cell membranes were resuspended in PBS buffer (10 mM NaPO4, $150 \mathrm{mM} \mathrm{NaCl}, 1 \mathrm{ml}$ per 50 $\mathrm{ml}$ original culture) containing $1 \% n$-dodecyl- $\beta$-D-maltoside (DDM) using sequentially an 18-G, 19-G, and 25-G needle. The suspension was incubated with pre-equilibrated Ni-NTA superflow resin (100 $\mu$ l per ml, Qiagen). The Ni-NTA superflow was washed twice with $20 \mathrm{mM}$ imidazole in PBS buffer containing $0.1 \%$ DDM, prior to elution with $250 \mathrm{mM}$ imidazole in PBS buffer containing $0.1 \%$ DDM.

Sodium dodecyl sulphate-polyacrylamide gel electrophoresis was performed as described in the literature (Sambrook and Russell 2001), using the Hoefer mighty small gel system for electrophoresis. Briefly, the resolving gel was prepared using $\sim 7 \mathrm{ml}$ of $10 \%$ acrylamide mix, National Diagnostics, $375 \mathrm{mM}$ Tris, $\mathrm{pH} 8.8$ and $0.1 \%$ SDS. Polymerisation was initiated by the addition of ammonium persulphate and $\mathrm{N}, \mathrm{N}, \mathrm{N}^{\prime}, \mathrm{N}^{\prime}$-tetramethylethylenediamine to final concentrations of 0.1 and $0.04 \%$, respectively. The stacking gel $(\sim 4 \mathrm{ml})$ consisted of $5 \%$ acrylamide mix, $127 \mathrm{mM}$ Tris, $\mathrm{pH} 6.8,0.1 \%$ SDS; polymerisation was initiated by addition of $0.1 \%$ ammonium persulphate and methods, without further purification. c Coomassie blue-stained SDS gel of purified P216L mutant from S2 cells. d p/RDS P216L mutant from $\mathrm{S} 2$ cells in the presence 1 and in the absence 2 of reducing agent. Note that the blot was performed using the solubilised membrane protein fraction without further purification

$\mathrm{N}, \mathrm{N}, \mathrm{N}^{\prime}, \mathrm{N}^{\prime}$-tetramethylethylenediamine to final concentrations of 0.1 and $0.01 \%$, respectively.

For the dimerisation experiments (Fig. 3b, d), membrane proteins were extracted from the lysed cells as described above without further purification by affinity chromatography. After removal of the cell debris and insoluble protein, $15 \mu \mathrm{l}$ of protein solution was mixed with $15 \mu \mathrm{l}$ of $2 \times$ SDS gel-loading buffer (100 mM Tris-Cl, $4 \%$ SDS, $0.2 \%$ bromophenol blue, and $20 \%$ glycerol). In the case of reducing conditions, $1 \% \beta$-mercaptoethanol was added to the loading buffer; samples were incubated for $30 \mathrm{~min}$ at $37^{\circ} \mathrm{C}$ and allowed to cool for $10 \mathrm{~min}$ at room temperature prior to loading on the gel. The non-reduced samples were loaded on the gel immediately after mixing with loading buffer. Analysis of purified protein with SDSPAGE (Figs. 3a, c, 4a, b) was performed as described for reducing conditions. Electrophoresis was performed at $100 \mathrm{~V}$ at room temperature for $\sim 2 \mathrm{~h}$ until the dye-front reached the bottom of the gel. Silver staining was performed according to the instructions provided in the PlusOne $^{\mathrm{TM}}$ silver staining kit (GE Healthcare). Coomassie staining was performed on a shaking platform for $1 \mathrm{~h}$ at room temperature using $10 \%$ glacial acetic acid, $45 \%$ methanol, $45 \%$ distilled water and $0.25 \%$ Coomassie Brilliant Blue R. Destaining was done using $7.5 \%$ glacial acetic acid and $10 \%$ methanol for $24 \mathrm{~h}$.

Proteins were transferred to PVDF membrane (Amersham) using a transfer unit for semi-dry transfer (Cleaver Scientific) according to the manufacturer's instructions. Western blots were probed with c-myc tag mouse monoclonal antibody (Cell Signaling), using anti-mouse HRP conjugated antibody as the secondary (Promega). Mass spectrometry analysis was performed on a an LC/MS system consisting of a 1,200 Series liquid chromatograph, an HPLC-Chip Cube MS interface and SL IT mass spectrometer (all Agilent Technologies, USA). The 

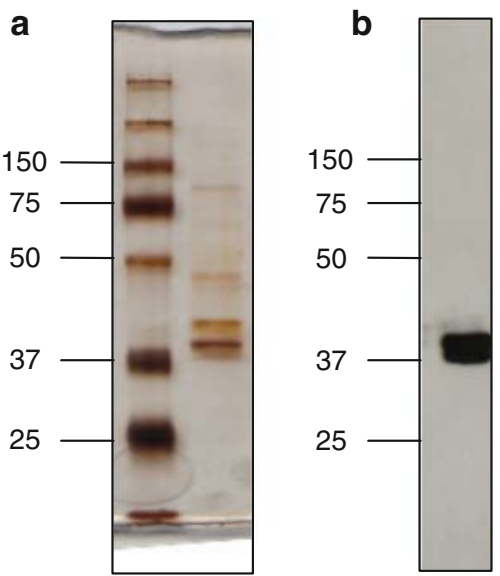

Fig. 4 a Silver-stained SDS gel of p/RDS from Pichia pastoris purified with Ni-NTA superflow agarose. b Western blot of p/RDS from $P$. pastoris purified with Ni-NTA superflow agarose. The blot was probed with monoclonal anti-c-myc antibody. Note that the purity in the case is less due to non-specific binding of yeast proteins to $\mathrm{Ni}-\mathrm{NTA}$ superflow agarose

chromatographic chip incorporated a 40-nl enrichment column, a $150 \mathrm{~mm} / 75-\mu \mathrm{m}$ analytical column packed with Zorbax 300SB-C18 5-mm particles and a nanospray needle. In-gel protein digests were adapted from the protocol by Shevchenko et al. (1996).

Circular dichroism

The CD spectrum of wild-type p/RDS and of the P216L mutant pRDS in $10 \mathrm{mM}$ Tris/HCl (pH 8.0) buffer with $1 \%$ DDM was acquired on a Model 400 Spectrometer (Aviv Biomedical Ltd.) using a strain-free certified 0.1 (mutant) or 1 (wild-type) $\mathrm{mm}$ SUPRASIL quartz rectangular demountable cell (Hellma UK) and as an average of 20 scans, at a scan rate of $1 \mathrm{~s}$, a temperature of $20^{\circ} \mathrm{C}$, a scan increment of $0.2 \mathrm{~nm}$ and a bandwidth of $1 \mathrm{~nm}$. The spectrum was background corrected by subtracting the CD spectrum of $10 \mathrm{mM}$ Tris/ $\mathrm{HCl}$ in $1 \%$ DDM acquired under identical conditions and spectral parameters as that of the sample. All CD spectra were acquired where the Model 400 dynode voltage was kept below 500 volts, as per specification. The $\mathrm{CD}$ spectrum was analysed with the CDSSTR (Sreerama and Woody 2000) and K2D (Andrade et al. 1993) programs using the Dichroweb server (Whitmore and Wallace 2004). Protein concentration was determined by quantitative amino acid analysis (PNAC Facility, Cambridge, UK) (wild-type protein) and by BCA assay (wild-type protein, P216L mutant).

Molecular modelling

Pictures of the protein models were constructed using the MOLMOL software (Koradi et al. 1996); secondary structures assignments were carried out using the Kabsch and Sander algorithm (Kabsch and Sander 1983). Secondary structure predictions were performed with the Jpred3 webserver (Cole et al. 2008). Protein threading was carried out using the TASSER software (Zhang 2008).

\section{Results}

$\mathrm{p} / \mathrm{RDSp}$ expression in $\mathrm{S} 2$ cells

Analysis by SDS-PAGE of the purified wild-type p/RDS and of the P216L mutant (Fig. 3a, c, respectively) shows a single band with an apparent molecular weight (AMW) of $\sim 37 \mathrm{kD}$ consistent with the apparent molecular weight of the monomeric protein reported in the literature (Goldberg et al. 2001), although some dimeric species can also be observed in the case of the mutant. The final purified protein yield was 50 and $220 \mu \mathrm{g}$ from $\sim 11$ of culture for the wild-type protein and the $\mathrm{P} 216 \mathrm{~L}$ mutant, respectively. The protein concentrations used for the $\mathrm{CD}$ experiments were 50 and $220 \mu \mathrm{g} / \mathrm{ml}$ as determined by BCA assay.

The identity of both the wild-type p/RDS and the P216L mutant was confirmed using Western blot probed with a HRP-conjugated V5 antibody with both proteins running as a single band at the appropriate size in the presence of reducing agent (see Fig. 3b, d, respectively). In addition, analysis by LC-MS/MS mass spectrometry of the wildtype protein identified seven human $\mathrm{p} / \mathrm{RDS}$ peptides after in-gel digestion (MASCOT score 243, cutoff value at $P<0.05$ was 52 in this search).

In vivo, $\mathrm{p} / \mathrm{RDS}$ forms tetrameric core complexes; the core complexes are linked by intermolecular disulphide bridges to form higher order oligomers, a process that is modulated by a close structural neighbour of peripherin/ RDS, ROM-1 (Goldberg et al. 2001; Loewen et al. 2001; Loewen and Molday 2000). The formation of these corecomplex linking disulphide bridges requires proximity of the sulphur atoms involved, which in turn requires correct folding of the protein. Therefore, the presence of disulphide-bonded dimers on SDS-PAGE is an indication that the protein is able to self-assemble and is correctly folded. To assess the ability for self-assembly of both wild-type and mutant P216L p/RDS, the electrophoretic mobility in the presence and in the absence of a reducing agent was analysed using SDS-PAGE. For both the wild-type and mutant protein, the Western blot of the reduced samples show a strong band at $37 \mathrm{kDa}$ corresponding to monomeric peripherin/rds. In the absence of $\beta$-mercaptoethanol, the $70-\mathrm{kDa}$ band corresponding to the dimeric species is the prominent species for both proteins (Fig. 3b, d, upper arrow). Furthermore, under non-reducing conditions, the monomer displays an increase in electrophoretic mobility relative to 
Fig. 5 a CD spectra of wildtype $\mathrm{p} / \mathrm{RDS}$ (red) and of the P216L mutant (blue). The wild type was measured in a $1-\mathrm{mm}$ cuvette, the mutant in a $0.1-\mathrm{mm}$ cuvette. b Same spectra as (a), but normalised to the same intensity. c Both proteins measured in 1-mm cuvette. Note that the spectrum of the wild-type protein could not be collected for wavelengths below $200 \mathrm{~nm}$ due to a decreased concentration
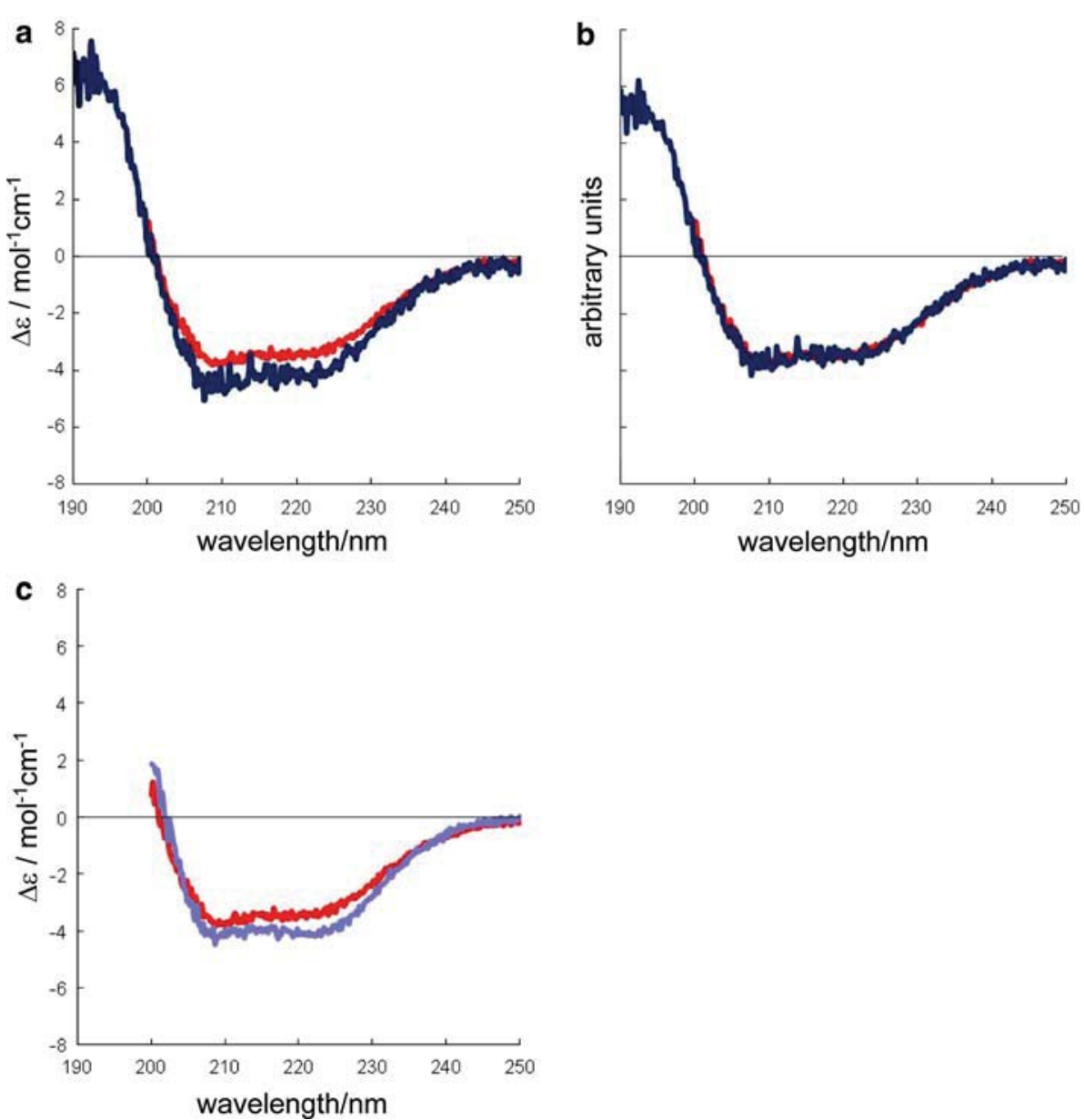

the monomer in the presence of $\beta$-mercaptoethanol under reducing conditions (Fig. $3 \mathrm{~d}$, b, lower arrow). The same pattern is observed for the $\mathrm{P} 216 \mathrm{~L}$ mutant, with the band corresponding to the monomer running at a slightly lower apparent molecular weight in the absence of reducing agent.

\section{p/RDSp expression in Pichia pastoris}

Analysis by SDS-PAGE of the purified wild-type p/RDS expressed in Pichia pastoris shows multiple bands after staining, with two bands (Fig. 4) corresponding to apparent molecular weights of $\sim 37$ and $\sim 40 \mathrm{kD}$ being the dominant species. The apparent molecular weight of $37 \mathrm{kD}$ is consistent with the apparent molecular weight of the monomer reported in the literature (Wrigley et al. 2002). The same two bands are present upon analysis with Western blot at apparent molecular weights of $\sim 37$ and $\sim 40 \mathrm{kD}$.

Structural characterisation of wild-type and mutant P216L p/RDS from S2 cells

Circular dichroism (CD) spectroscopy was used to characterise the secondary structure of $\mathrm{p} / \mathrm{RDSp}$ in $n$-dodecyl- $\beta$ D-maltoside (DDM). P/RDSp shows bands at $\sim 208$ and at $\sim 222 \mathrm{~nm}$, indicative of $\alpha$-helical structure (Fig. 5a). The intensity of the $\mathrm{CD}$ spectrum of the $\mathrm{P} 216 \mathrm{~L}$ mutant is slightly increased as compared to that of the wild type. The shape of the P216L mutant is similar to that of the wild-type protein (Fig. 5b, c), with bands at $\sim 208$ and $\sim 222 \mathrm{~nm}$ indicating the presence of significant $\alpha$-helical structure. Further comments are included in the discussion.

\section{Discussion}

Expression of recombinant peripherin/RDS in S2 cells and in Pichia pastoris

Our results clearly demonstrate the expression of both the wild-type and the mutant protein in S2 cells, and expression of the wild-type protein in the methylotrophic yeast Pichia pastoris. Expression levels in S2 cells are sufficient for a structural characterisation with circular dichroism spectroscopy (total yield of $\sim 50 \mu \mathrm{g} / \mathrm{l}$ culture for the wild type and $\sim 220 \mu \mathrm{g} / \mathrm{l}$ culture for the mutant protein). However, in order to perform high-resolution structural determination using NMR or X-ray crystallography, and also for alternative techniques of secondary structure 
determination such as FTIR-spectroscopy, larger protein quantities are required than those obtained here. When scaling up the production of recombinant protein in cultured insect cells, the cost effectiveness of this system should be considered, since the procedure for growing insect cells in tissue culture is labour intensive, and the chemicals are expensive compared to those required for protein expression in bacteria and yeast. For this reason, the demonstrated expression of p/RDS in Pichia pastoris is a significant advance. Preliminary data from experiments in small-scale bioreactors indicate expression levels of $\sim 0.3 \mathrm{mg} / \mathrm{l}$, which is comparable to the per liter expression of the protein in $\mathrm{S} 2$ cells. This protein could be purified to the same degree as the protein from S2 cells using sequentially Ni-NTA and cmyc agarose. Using optimised protocols for culturing the yeast, we believe that the protein expression can be increased even further.

Self-assembly of wild-type and P216L mutant peripherin/RDS from $\mathrm{S} 2$ cells

The presence of a single band for both the purified wildtype $\mathrm{p} / \mathrm{RDS}$ and for the $\mathrm{P} 216 \mathrm{~L}$ mutant shows that pure protein was obtained after the two-step purification approach using sequentially Ni-NTA superflow and V5 agarose. No bands are observed at higher molecular weight, indicating that the protein does not form non-specific aggregates when exposed to the reducing conditions used for SDS-PAGE. The Western blot of both wild-type and mutant peripherin/RDS in the presence of reducing agent shows a single band at the apparent molecular weight of the protein, although some higher molecular weight species are also observed in the case of the mutant protein. However, it is clear that in the absence of reducing agent for both wild-type and mutant, the relative amount of dimer is much increased.

Furthermore, in the absence of reducing agent, the apparent molecular weight of both the monomeric mutant and the wild-type protein decreases slightly, which could suggest a more compact structure of the monomer. Possibly, in the absence of reducing agent some disulphide bridges remain intact, resulting in retention of residual structure in the presence of SDS. Also, for both wild-type and mutant protein, the dimer is the dominant species, highlighting the capacity of both wild-type and P216L mutant protein to self-assemble. Although SDS-PAGE can give rise to nonspecific aggregation, the formation of specific dimers is well documented for p/RDS [see, for instance, (Loewen and Molday 2000)]. Therefore, our observations suggest that both the wild-type and the mutant protein are correctly folded, and that the P216L mutation has no strong disruptive effect on the structure of the LEL domain.

\section{Expression of p/RDS in Pichia pastoris}

The silver-stained gel after single-step purification with Ni-NTA resin shows a number of non-specific protein bands, indicating binding of protein other than hexahistidine-tagged $\mathrm{p} / \mathrm{RDS}$. Non-specific protein binding to nickel affinity chromatography is a common problem for recombinant protein expressed in eukaryotes as compared to prokaryotes since the fraction of histidine-rich proteins is higher, and the total number of proteins is larger.

The Western blot of p/RDS from Pichia Pastoris shows a band at an apparent molecular weight of $\sim 37 \mathrm{kD}$. The second band at $\sim 40 \mathrm{kD}$ could be indicative of extra glycosylation, which has been observed before for recombinant glycoproteins expressed in Pichia Pastoris [(Ruitenberg et al. 2001)]. Interestingly, also when $\mathrm{p} / \mathrm{RDS}$ is expressed in COS cells, it migrates as a doublet on SDS-PAGE, which was ascribed to the presence of both glycosylated and nonglycosylated forms (Muller-Weeks et al. 2002), suggesting that also in the present study at least part of the protein expressed in Pichia is glycosylated.

\section{Implications of the $\mathrm{CD}$ experiments}

The CD experiments presented here allow for a direct assessment of the effect of a pathogenic point mutation on the secondary structure of peripherin/RDS. Comparison of the shape of the CD spectra (Fig. 5a) suggests that the secondary structure of the pathogenic P216L mutant is similar to that of the wild-type protein. To illustrate this, the spectra were normalised to the same intensity and superimposed in Fig. 5b. Clearly the shape of the spectra is almost identical for wavelengths between 200 and $250 \mathrm{~nm}$, indicating that the structure of the mutant is similar to that of the wild-type protein. This is further illustrated in Fig. 5c, which shows the CD spectra of the wild type and the mutant measured in the same cuvette. To further examine this idea, a secondary structure analysis was used to calculate the fraction of $\alpha$-helix and $\beta$-sheet in both mutant and wild-type protein. Table 1 shows that the fraction of $\alpha$-helix is increased for the mutant, whereas the fraction of $\beta$-sheet is similar. However, since the shape of the CD spectra is similar, these differences arise from small differences in intensity of the spectrum that can strongly influence the outcome of a secondary structure calculation based on CD data. It is well documented that the magnitude of the spectrum is affected by uncertainties in protein concentration and in cuvette path length (McPhie 2008). Most likely, in the present study, such factors also affected the outcome of the secondary structure analysis. Therefore, we conclude that the effect of the pathogenic mutation on the secondary structure is small based on the similarity in spectral shape and on the similar biochemical behaviour of 
Table 1 Percentages of $\alpha$-helix, $\beta$-sheet and coil for the wild-type protein and for the P216L mutant

\begin{tabular}{llllllc}
\hline Protein & $\begin{array}{l}\text { Type of cuvette } \\
\text { used }(\mathrm{mm})\end{array}$ & $\begin{array}{l}\text { Algorithm } \\
\text { used/dataset } \\
\text { (if applicable) }\end{array}$ & $\begin{array}{l}\text { Fraction } \alpha \text {-helix/nr } \\
\text { of residues } \\
\text { in a-helix }\end{array}$ & $\begin{array}{l}\text { Fraction } \beta \text {-sheet/nr } \\
\text { of residues } \\
\text { in } \beta \text {-sheet }\end{array}$ & $\begin{array}{l}\text { Fraction coil/nr } \\
\text { residues in coil/ } \\
\text { unordered }\end{array}$ & $\begin{array}{l}\text { NRSMD } \\
\text { p/RDS, wild type }\end{array}$ \\
p/RDS, P216L mutant & 1 & K2D/NA & $0.29 / 109$ & $0.16 / 60$ & $0.54 / 208$ & 0.069 \\
p/RDS, P216L mutant & 0.1 & K2D/NA & $0.55 / 207$ & $0.13 / 49$ & $0.33 / 121$ & 0.105 \\
p/RDS, P216L mutant & 0.1 & K2D/NA & $0.57 / 215$ & $0.08 / 30$ & $0.35 / 132$ & 0.039 \\
\hline
\end{tabular}

Note that the fractions of turn and unordered were summed up to give the fraction of coil in the case of the CDSSTR analysis. The upper two rows are the results from the $\mathrm{K} 2 \mathrm{D}$ analysis in 1-mm cuvette; the two bottom rows are results from the analysis done on data measured in the $0.1 \mathrm{~mm}$ cuvette

the mutant to the wild-type protein upon analysis with SDS-PAGE.

Although it has been shown that the P216L mutation causes photoreceptor degeneration, it is properly trafficked to the outer segment (Loewen et al. 2003). From previous studies it is known that an additional glycosylation site is created by this mutation (Wrigley et al. 2002), and it was suggested that the attachment of two bulky carbohydrate chains to the protein may result in an altered conformation in the protein. The SDS-PAGE analysis presented here indicates that the glycosylation status of wild-type $\mathrm{p} / \mathrm{RDS}$ and the P216L mutant expressed in $\mathrm{S} 2$ cells is the same; therefore, it is not possible to completely test this hypothesis. However, the results presented here show that the secondary structure is largely unaffected by the P216L mutation. Possibly, the fold of the LEL-domain (i.e., the arrangement of the secondary structure elements) is influenced by the mutation instead, an effect that could be further exacerbated by differences in glycosylation status for this particular mutant (Wrigley 2002, p. 288).

We note, however, that previous studies have shown that the insertion of a FLAG-epitope cassette between residues I227 and T228, close to the P216, results in a change in sedimentation behaviour indicating a disruption in subunit assembly without impairing protein folding, whereas insertion of the same FLAG epitope at most other sites did not influence its sedimentation profile (Goldberg et al. 2001).

Towards a three-dimensional structure of the LEL domain

To fully evaluate the implications of our CD data, we compared the fractions of secondary structure of wild-type $\mathrm{p} / \mathrm{RDS}$ to the topology model from our earlier work (Wrigley et al. 2002). Neither the V5 epitope nor the hexahistidine tag is expected to contribute secondary structure (Li et al. 2006; Narayana et al. 1997). Also, since both tags are located at the intrinsically disordered C-terminal domain (Edrington et al. 2007), they are not expected to interfere with the structure or function of $\mathrm{p} / \mathrm{RDS}$.
The CD secondary structure analysis shows that the fraction of $\alpha$-helix is $0.29-0.57$, the equivalent of 109-215 amino acid residues. The $\alpha$-helical residues are mostly accounted for by the four transmembrane helices that together make up $\sim 81 \alpha$-helical residues. For the tetraspanin CD81 it has been shown that the LEL domain is predominantly $\alpha$-helical (Kitadokoro et al. 2001). Based on homology to the LEL domain of tetraspanin CD81, it is likely that for peripherin/RDS, a large portion of the nontransmembrane $\alpha$-helical residues is located in this LELdomain. Also, part of the observed $\beta$-sheet structure is probably located in the LEL domain.

For comparison, a structure prediction of the $\mathrm{p} / \mathrm{RDS}$ LEL domain was performed using Jpred 3 software (Cole et al. 2008; Fig. 6) for secondary structure prediction and using the computer program TASSER (Threading ASSEmbly Refinement) (Zhang and Skolnick 2004) for three-dimensional structure prediction. Jpred 3 predicts with high confidence the $\alpha$-helix between amino acid residues 123-138 (conserved tetraspanin helix A, Seigneuret 2006), between residues 151-162 (helix B) and between residues 251-259 (helix E) with patches of $\alpha$-helix and $\beta$-sheet in the region between.

Two three-dimensional models, depicted in Fig. 7 (left and middle panel), are returned by the TASSER threading software with a TM score greater than threshold (the TM scores for these models are 0.73 and 0.58 , respectively: a TM score $>0.5$ indicates a model of correct topology and a TM score $<0.17$ means random similarity, Zhang and Skolnick 2004). For comparison, the structure of the CD81 LEL domain is also depicted in Fig. 7 (right panel). Although the three-dimensional structure of the two TASSER models is different, the secondary structure largely overlaps, with $\alpha$-helices A, B and E present in approximately the same places as for the $2 \mathrm{D}$ model. The main differences with the $2 \mathrm{D}$ model is that the $\beta$-sheet content is zero, and the fraction of $\alpha$-helix is increased. In summary, our findings from the CD experiments, which suggest the presence of a $\beta$-sheet in the p/RDS variable region, are qualitatively in better agreement with the 
Fig. 6 Secondary structure prediction using JPRED3 software for the $\mathrm{p} / \mathrm{RDS}$ LEL domain. Helices are indicated as purple cylinders; $\mathrm{b}$-sheet is indicated as yellow arrows. The number above the amino acid sequence indicates the position of the residue in $\mathrm{p} / \mathrm{RDS}$; the number below indicates the confidence. Cystein residues are highlighted in yellow; hydrophobic residues are indicated by squares. The proline residues at position 216 are highlighted with a blue circle

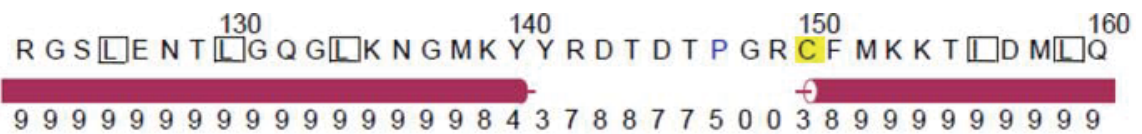

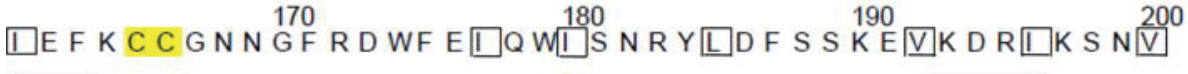
8800577776656700001301125677770000000777

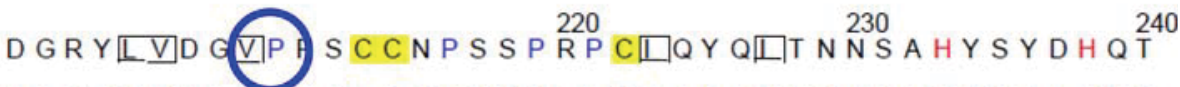
7777777776414577777641046777777777676777 E EL L N L WV R G C 250 A ALL $S$ Y Y S 260 7400000000078999999877100000

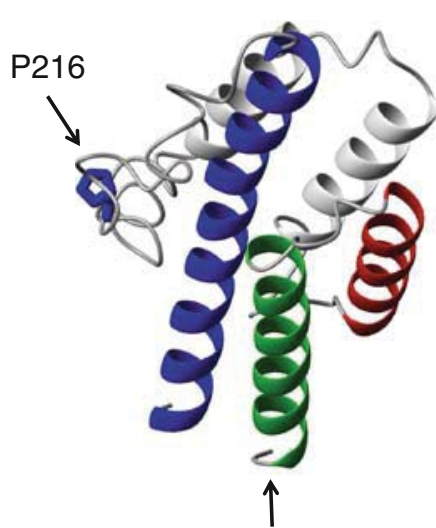

R123

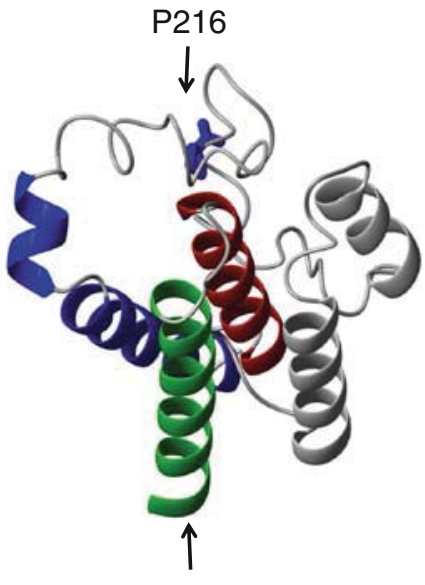

R123

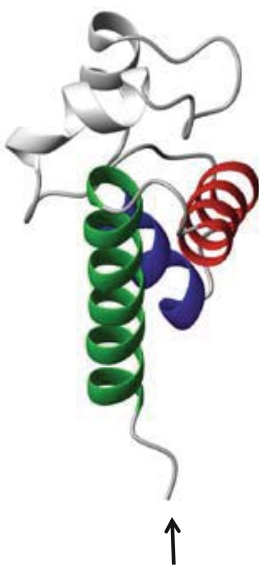

F113
Fig. 7 Left and middle panel: best scoring models (TM values are 0.73 and 0.58 , respectively) of the p/RDS LEL domain calculated using the I-TASSER software for structure prediction. Right panel: tetraspanin CD81. Helices are represented as coloured ribbons. Conserved tetraspanin helices A, B and E are depicted in green, red

secondary structure predictions, which show patches of $\beta$-sheet in the LEL domain, than with the TASSER models, which show a mixture of $\alpha$-helix and coil, possibly because the TASSER software tries to accommodate the model to the only known three-dimensional tetraspanin structure. Quantitatively, our CD data suggest that the amount of $\beta$-sheet is even greater than indicated by the secondary structure predictions, which show only $\sim 7$ residues in a $\beta$-sheet conformation in the LEL domain.

\section{Concluding remarks}

We have demonstrated expression of peripherin/RDS in both S2 cells and in the methylotrophic yeast Pichia and blue, respectively. Helices in the tetraspanin variable domain are indicated in white. Coil domains are represented as grey tubes. The $\mathrm{N}$ terminal residue (R123 for p/RDS, F113 for CD81) is indicated with an arrow. The proline residue at position 216 is indicated in blue

Pastoris. P/RDS from S2 cells was further characterised, and the effect of the pathogenic P216L mutation was evaluated. The structural effect of the pathogenic mutation is small, indicating that the mutation might have an indirect effect, without disturbing its secondary structure. The main implication of our CD experiments is the presence of an unexpected fraction of $\beta$-sheet, which is tentatively assigned to the variable region of the LEL domain. We are currently expressing the LEL domain as a separate soluble protein in order to confirm the presence of a $\beta$-sheet in this domain and to assess its functionality.

Acknowledgments We acknowledge the Transfer of Knowledge Scheme (Marie-Curie) and IRCSET for funding. The authors acknowledge Dr. Gemma Kinsella for critically reading the manuscript and useful discussion. 


\section{References}

Andrade MA, Chacon P, Merelo JJ, Moran F (1993) Evaluation of proteins from UV circular dichroism using an unsupervised learning nearal network. Prot Eng 6:383-390

Cole C, Barber JD, Barton GJ (2008) The Jpred 3 secondary structure prediction server. Nucleic Acids Res 36:W197-W201

Edrington TC, Lapointe VR, Yeagle PL, Gretzula CL, BoeszeBattaglia K (2007) Peripherin-2: an intracellular analogy to viral fusion proteins. Biochemistry 46:3605-3613

Goldberg AFX, Fales LM, Khattree N, Hurley JB (2001) Folding and subunit assembly of photoreceptor peripherin/rds is mediated by determinants within the extracellular/intradiskal EC2 domain: implications for heterogeneous molecular pathologies. J Biol Chem 276:42700-42706

Hemler ME (2003) Tetraspanin proteins mediate cellular penetration invasion and fusion events, and define a novel type of membrane microdomain. Annu Rev Cell Dev Biol 19:397-422

Hemler ME (2005) Tetraspanin functions and associated microdomains. Nat Rev Mol Cell Biol 6:801-811

Invitrogen (1997) A manual of methods for expression of recombinant proteins in Pichia pastoris. In: Corporation I (ed) San Diego

Invitrogen (2003) Drosophila expression system. In: Corporation I (ed) San Diego

Jamshad M, Rajesh S, Stamataki Z, McKeating JA, Dafforn T, Overduin M, Bill RM (2008) Structural characterization of recombinant human CD81 produced in Pichia pastoris. Prot Expr Purif 57:206-216

Kabsch W, Sander C (1983) Dictionary of protein secondary structure: pattern recognition of hydrogen-bonded and geometrical features. Biopolymers 22:2577-2637

Kitadokoro K, Bordo D, Galli G, Petracca R, Falugi F, Abrignani S, Grandi G, Bolognesi M (2001) CD81 extracellular domain 3D structure: insight into the tetraspanin superfamily structural motifs. EMBO J 20:12-18

Koradi R, Billeter M, Wuthrich K (1996) MOLMOL: a program for display and analysis of macromolecular structures. J Mol Graph 14:51-55

Lazo PA (2007) Functional implications of tetraspanin proteins in cancer biology. Cancer Sci 98:1666-1677

Levy S, Todd SC, Maecker HT (1998) CD81 (TAPA-1): a molecule involved in signal transduction and cell adhesion in the immune system. Annu Rev Immunol 16:89-109

Li T, Chen X, Garbutt KC, Zhou P, Zheng N (2006) Structure of DDB1 in complex with a paramyxovirus $\mathrm{V}$ protein: viral hijack of a propeller cluster in ubiquitin ligase. Cell 13:105-117

Loewen CJR, Molday RS (2000) Disulfide-mediate oligomerization of peripherin/RDS and Rom-1 in photoreceptor disk membranes. J Biol Chem 275:5370-5378

Loewen CJ, Moritz OL, Molday RS (2001) Molecular characterization of peripherin- 2 and Rom-1 mutants responsible for digenic retinitis pigmentosa. J Biol Chem 276:22388-22396

Loewen CJ, Moritz OL, Tam BM, Papermaster DS, Molday RS (2003) The role of subunit assembly in peripherin-2 targeting to rod photoreceptor disk membranes and retinitis pigmentosa. Mol Biol Cell 14:3400-3413

McPhie P (2008) Concentration independent estimation of protein secondary structure by circular dichroism; a comparison of methods. Anal Biochem 375:379-381
Molday RS, Hicks D, Molday L (1987) Peripherin. A rim specific membrane protein of rod outer segment discs. Invest Ophthalmol Vis Sci 28:50-61

Muller-Weeks S, Boesze-Battaglia K, Fitzgerald C (2002) Deletional analysis of the rod photoreceptor cell peripherin/RDS carboxyterminal region. Exp Eye Res 75:143-154

Narayana N, Cox S, Shaltiel S, Taylor SS, Xuong N-h (1997) Crystal structure of a polyhistidine-tagged recombinant catalytic subunit of camp-dependent protein kinase complexed with the peptide inhibitor PKI(5-24) and adenosine. Biochemistry 36:4438-4448

Palczewski K (2006) G protein-coupled receptor rhodopsin. Annu Rev Biochem 75:743-767

Ruitenberg KM, Gilkerson JR, Wellington JE, Love DN, Whalley JM (2001) Equine herpesvirus 1 glycoprotein D expressed in Pichia pastoris is hyperglycosylated and elicits a protective immune response in the mouse model of EHV-1 disease. Virus Res 79:125-135

Sambrook J, Russell DW (2001) Molecular cloning, a laboratory manual, vol 3, 3rd edn. Cold spring harbor laboratory press, New York

Seigneuret M (2006) Complete predicted three-dimensional structure of the facilitator transmembrane protein and hepatitis $\mathrm{C}$ virus receptor CD81: conserved and variable domains in the tetraspanin superfamily. Biophys J 90:212-217

Shastry BS (1994) Retinitis pigmentosa and related disorders: phenotypes of rhodopsin and peripherin/RDS mutatations. Am J Med Genet 52:467-474

Shevchenko A, Wilm M, Vorm O, Mann M (1996) Mass spectrometry sequencing of protein from silver-stained polyacrylamide gels. Anal Chem 68:850-858

Sreerama N, Woody R (2000) Estimation of protein secondary structure from CD spectra: comparison of CONTIN, SELCON, and CDSSTR methods with an expanded reference set. Anal Biochem 282:252-260

Takayama H, Chelikani P, Reeves PJ, Zhang S, Khorana HG (2008) High-level expression, single-step iummunoaffinity purification and characterization of human tetraspanin membrane protein CD81. PLoS One 3:1-7

Whitmore L, Wallace BA (2004) DICHROWEB, an online server for protein secondary structure analyses from circular dichroism spectroscopic data. Nucleic Acids Res 32:W668-W673

Wrigley JDJ, Ahmed T, Nevett CL, Findlay JBC (2000) Peripherin/ $r d s$ influences membrane vesicle morphology. J Biol Chem 275:13191-13194

Wrigley JD, Nevett CL, Findlay JBC (2002) Topological analysis of peripherin/RDS and abnormal glycosylation of the pathogenic Pro $^{216}$ Leu mutation. Biochem J 368:649-655

Zhang Y (2008) I-TASSER server for protein 3D structure prediction. BMC Bioinformatics 9

Zhang Y, Skolnick J (2004) Scoring function for automated assessment of protein structure template quality. Proteins $57: 702-710$

Zhang J, Randall G, Higginbottom A, Monk P, Rice CM, McKeating JA (2004) CD81 is required for hepatitis C virus glycoproteinmediated viral infection. J Virol 78:1448-1455 